\title{
Screening of gene signatures for rheumatoid arthritis and osteoarthritis based on bioinformatics analysis
}

\author{
PEIHENG HE ${ }^{*}$, ZIJI ZHANG* , WEIMING LIAO, DONGLIANG XU, MING FU and YAN KANG \\ Department of Joint Surgery, The First Affiliated Hospital of Sun Yat-sen University, \\ Guangzhou, Guangdong 510080, P.R. China
}

Received June 16, 2015; Accepted May 5, 2016

DOI: $10.3892 / \mathrm{mmr} .2016 .5423$

\begin{abstract}
The current study aimed to identify gene signatures during rheumatoid arthritis (RA) and osteoarthritis (OA), and used these to elucidate the underlying modular mechanisms. Using the Gene Expression Omnibus database, the present study obtained the GSE7669 mRNA expression microarray data from RA and OA synovial fibroblasts ( $n=6$ each). The differentially expressed genes (DEGs) in RA synovial samples compared with OA samples were identified using the Linear Models for Microarray Analysis package. The Gene Ontology and Kyoto Encyclopedia of Genes and Genomes pathway enrichment analyses were performed using the Database for Annotation Visualization and Integrated Discovery. A protein-protein interaction network was constructed and the modules were further analyzed using the Molecular Complex Detection plugin of Cytoscape. A total of 181 DEGs were identified by comparing RA and OA synovial samples (96 up- and 85 downregulated genes). The significant DEGs in module 1, including collagen, type I, $\alpha 1$ (COL1A1), COL3A1, COL4A1 and COL11A1, were predominantly enriched in the extracellular matrix (ECM)-receptor interaction and focal adhesion pathways. Additionally, significant DEGs in module 2, including radical $\mathrm{S}$-adenosyl methionine domain containing 2 (RSAD2), 2'-5'-oligoadenylate synthetase 2 (OAS2), myxovirus (influenza virus) resistance 1 (MX1) and ISG15 ubiquitin-like modifier (ISG15), were predominantly associated with immune function pathways. In conclusion, the present study indicated that RSAD2, OAS2, MX1 and ISG15 may be notable gene signatures in RA development via regulation of the immune response. COL3A1, COL4A1, COL1A1 and COL11A1 may be important gene signatures in $\mathrm{OA}$
\end{abstract}

Correspondence to: Dr Yan Kang, Department of Joint Surgery, The First Affiliated Hospital of Sun Yat-sen University, 58 Zhongshan 2nd Road, Yuexiu, Guangzhou, Guangdong 510080, P.R. China E-mail: naykang@163.com

*Contributed equally

Key words: rheumatoid arthritis, osteoarthritis, differential expression genes, functional enrichment analysis, module analysis development via involvement in the pathways of ECM-receptor interactions and focal adhesions.

\section{Introduction}

Rheumatoid arthritis (RA) is a chronic inflammatory disease which can lead to progressive disability, early mortality and other systemic complications (1). RA is incurable, has high socioeconomic costs and severely reduces the quality of life of patients (2). Osteoarthritis (OA) is the most common joint disease; it causes pain, disability and the loss of joint function (3). OA is also a chronic and incurable illness that lacks effective treatment strategies (4). Thus, advances in the understanding of the molecular mechanisms underlying these two diseases may lead to the development of novel therapeutic strategies.

RA is characterized by autoimmune and synovial inflammation, the destruction of multiple joints and the formation of pannus (5). OA is characterized by an inflammatory response and progressive breakdown of the articular cartilage of the joint (6). Genetic factors have been implicated in the development of these diseases $(7,8)$. The human leukocyte antigen molecules and their relevant immunological pathways have been associated with RA pathogenesis (9). Sun et al (10) demonstrated that paired immunoglobin-like type receptor $\alpha$ was associated with inflammatory cell infiltration and was elevated in the synovial tissue from mice with RA. In addition, a genome-wide association and functional study suggests that DOT1-like histone H3K79 methyltransferase is associated with cartilage thickness and hip OA (11). Valdes et al (12) also confirmed that genetic variation in the SMAD family member 3 gene may result in the progression of hip and knee OA. Together, these findings indicate the importance of genetic mechanisms in the pathogenesis of RA and OA. Despite previous progress, the gene signatures associated with the pathogenesis of RA and OA remain unknown, and reliable predictive biomarkers for prognosis and treatment are lacking.

Microarray analyses have been increasingly used to identify disease-associated genes and pathways for elucidation of the molecular mechanisms of RA and OA $(13,14)$. In a previous study, the GSE7669 microarray data was used to analyze differentially expressed genes (DEGs) between RA and OA using a gene co-expression network (15), or to screen candidate genes associated with RA by investigating core 
Table I. The top 10 significantly enriched GO biological progress terms.

\begin{tabular}{clcc}
\hline GO term & \multicolumn{1}{c}{ Description } & No. enriched genes & P-value \\
\hline Upregulated & Cell adhesion & 24 & $1.35 \times 10^{-11}$ \\
0007155 & Biological adhesion & 24 & $1.39 \times 10^{-11}$ \\
0022610 & Cell surface receptor linked signal transduction & 19 & $2.74 \times 10^{-2}$ \\
0007166 & Extracellular structure organization & 17 & $1.99 \times 10^{-15}$ \\
0043062 & Extracellular matrix organization & 16 & $4.32 \times 10^{-17}$ \\
0030198 & Blood vessel development & 12 & $2.48 \times 10^{-7}$ \\
0001568 & Vasculature development & 12 & $3.16 \times 10^{-7}$ \\
0001944 & Skeletal system development & 12 & $3.32 \times 10^{-6}$ \\
0001501 & Collagen fibril organization & 10 & $6.38 \times 10^{-14}$ \\
0030199 & Response to organic substance & 10 & $3.01 \times 10^{-2}$ \\
0010033 & & & \\
Downregulated & Regulation of cell death & 9 & $4.73 \times 10^{-2}$ \\
0010941 & Response to hormone stimulus & 7 & $9.50 \times 10^{-3}$ \\
0009725 & Response to endogenous stimulus & 7 & $1.49 \times 10^{-2}$ \\
0009719 & Positive regulation of cell death & 7 & $2.05 \times 10^{-2}$ \\
0010942 & Regulation of cell growth & 6 & $2.71 \times 10^{-3}$ \\
0001558 & Regulation of growth & 6 & $2.67 \times 10^{-2}$ \\
0040008 & Response to virus & 5 & $2.07 \times 10^{-3}$ \\
0009615 & Negative regulation of cell differentiation & 5 & $2.22 \times 10^{-2}$ \\
0045596 & Cellular response to hormone stimulus & 4 & $2.82 \times 10^{-2}$ \\
0032870 & Anion transport & 4 & $3.39 \times 10^{-2}$ \\
0006820 & & &
\end{tabular}

GO, gene ontology.

and periphery interaction structures (16). By contrast, the current study used this microarray data and comprehensive bioinformatics methods to identify DEGs in synovial RA samples compared with OA samples. Additionally, the present study performed functional enrichment analysis for DEGs and functional module analysis of the protein-protein interaction (PPI) network. The current study aimed to identify important disease-associated genes and the molecular mechanisms involved in RA and OA.

\section{Materials and methods}

Affymetrix microarray data. The GSE7669 gene expression profile deposited by Pohlers et al (17) was downloaded from Gene Expression Omnibus (www.ncbi.nlm.nih.gov/geo/), which was based on the platform of Affymetrix Human Genome U95 Version 2 Array (Affymetrix, Inc., Santa Clara, CA, USA). This dataset included the gene expression profiles from the synovial fibroblasts of 6 patients with RA and 6 patients with $\mathrm{OA}$.

Data preprocessing and DEG screening. All the raw expression data was preprocessed using the Affymetrix package (18) in R (cran.at.r-project.org) and Bioconductor (www.bioconductor.org), and the normalization was performed using the robust multiarray average algorithm (19). The gene expression matrix of samples was acquired.
DEGs in RA synovial samples compared with OA samples were identified using the Linear Models for Microarray Analysis (Limma; www.bioconductor. org/packages/release/bioc/html/limma.html) package (20) in $\mathrm{R} /$ Bioconductor. $\mathrm{t}$-test in the Limma package was used to analyze the P-value of each gene symbol. Only DEGs with $\mathrm{P}<0.05$ and $\log 2$ fold changel $>0.5$ were considered to indicate a statistically significant difference.

Functional enrichment analysis of DEGs. Gene Ontology (GO; www.geneontology.org) (21) is widely used in biology for the collation of large-scale gene lists, including biological process (BP) ontology. The Kyoto Encyclopedia of Genes and Genomes (KEGG; www.genome.ad.jp/kegg) (22) is used for extracting the pathway information from molecular interaction networks. To understand the biological significance of DEGs, GO BP enrichment analysis and KEGG pathway analysis were performed using the Database for Annotation Visualization and Integrated Discovery (DAVID; david. abcc.ncifcrf.gov) online tool (23). The $\mathrm{P}<0.05$ in the hypergeometric test and gene count $>2$ were defined as the cut-off values.

PPI network construction. The Search Tool for the Retrieval of Interacting Genes (STRING) (24) is a database providing information on experimentally verified and predicted protein interactions by calculating their combined score. Based on 
Table II. The significantly enriched KEGG pathways .

\begin{tabular}{|c|c|c|c|}
\hline KEGG pathway term & Description & No. enriched genes & P-value \\
\hline \multicolumn{4}{|l|}{ Upregulated } \\
\hline hsa04512 & Extracellular matrix-receptor interaction & 11 & $3.77 \times 10^{-10}$ \\
\hline hsa04510 & Focal adhesion & 13 & $1.60 \times 10^{-8}$ \\
\hline hsa04350 & Transforming growth factor- $\beta$ signaling pathway & 4 & $2.84 \times 10^{-2}$ \\
\hline \multicolumn{4}{|l|}{ Downregulated } \\
\hline hsa04350 & Transforming growth factor- $\beta$ signaling pathway & 4 & $1.28 \times 10^{-2}$ \\
\hline hsa04270 & Vascular smooth muscle contraction & 4 & $2.51 \times 10^{-2}$ \\
\hline
\end{tabular}

KEGG, Kyoto Encyclopedia of Genes and Genomes.

Table III. The top 20 differentially expressed genes with higher connectivity degree in the protein-protein interaction network.

\begin{tabular}{lc}
\hline Gene & Degree \\
\hline Collagen, type I, $\alpha$ 1 & 28 \\
Collagen, type I, $\alpha$ 2 & 27 \\
Collagen, type III, $\alpha$ 1 & 27 \\
Collagen, type IV, $\alpha$ 2 & 22 \\
Integrin subunit $\alpha$ 1 & 17 \\
Collagen, type IV, $\alpha$ 1 & 17 \\
Biglycan & 17 \\
Collagen, type XI, $\alpha$ 1 & 16 \\
Versican & 15 \\
Collagen, type IV, $\alpha$ 2 & 15 \\
Secreted protein, acidic, cysteine-rich & 15 \\
Periostin, osteoblast specific factor & 15 \\
Elastin & 13 \\
Cadherin 11 & 12 \\
Interferon induced protein & \\
with tetratricopeptide repeats 1 & 12 \\
Fibromodulin & 12 \\
Collagen, type XIV, $\alpha$ 1 & 12 \\
Interferon induced protein & 11 \\
with tetratricopeptide repeats 3 & 11 \\
Collagen, type XV, $\alpha$ 1 & 11 \\
Collagen, type XVI, $\alpha$ 1 & \\
\hline
\end{tabular}

the information of the STRING database, DEGs with the combined protein interaction score $>0.4$ were selected and used to construct a PPI network in the present study. Hub proteins (25) in the PPI network were identified based on connectivity degree analysis. The PPI network was visualized using Cytoscape software (www.cytoscape.org) (26).

Functional module analysis of PPI network. The functional modules of the PPI network were subsequently identified using the Molecular Complex Detection (MCODE) (27) plugin of Cytoscape. The parameters were set as follows: Degree cut-off, 2; node score cut-off, 0.2; K-core, 2; and max depth, 100. Functional enrichment analyses for DEGs in functional modules with higher degree and node scores were subsequently performed using the DAVID tool. $\mathrm{P}<0.05$ and gene count $>2$ were considered to indicate a statistically significant difference.

\section{Results}

DEG screening. Comparing OA and RA samples, a total of 181 DEGs were obtained, in which 96 genes were upregulated in RA and downregulated in OA samples, and 85 genes were downregulated in RA and upregulated in OA samples.

Functional enrichment analyses. The GO BP terms and KEGG pathway analyses were conducted for functional annotation of the DEGs. The top 10 GO BP terms are presented in Table I. The results demonstrated that the upregulated genes were significantly enriched in functions associated with cell adhesion, biological adhesion and extracellular matrix (ECM) organization. Downregulated genes were predominantly associated with the regulation of cell death, and responses to hormone and endogenous stimuli.

In addition, 3 and 2 KEGG pathways were significantly enriched by up- and downregulated DEGs, respectively (Table II). The enriched KEGG pathways for the upregulated genes were ECM-receptor interactions, focal adhesions and the TGF- $\beta$ signaling pathway, whereas the pathways enriched by the downregulated genes included the TGF- $\beta$ signaling pathway and vascular smooth muscle contraction (Table II).

PPI network analysis. Based on the information of the STRING database, a total of 343 protein interactions with combined scores $>0.4$ were included in the PPI network (Fig. 1). The top 20 hub proteins were identified according to connectivity degree (Table III), including collagen type I $\alpha 1$ (COL1A1), COL1A2, COL3A1, COL4A2, integrin $\alpha 1$, COL4A1, biglycan and COL11A1. Notably, in addition to interferon-induced protein with tetratricopeptide repeats 3 , these hub nodes were upregulated and the majority of them are collagen proteins.

Functional module analysis of PPI network. In order to improve analysis of the PPI network, 5 modules were detected using the MCODE plugin. Only 2 module scores were $>10$ and the scores of other modules were not $>3.5$. 


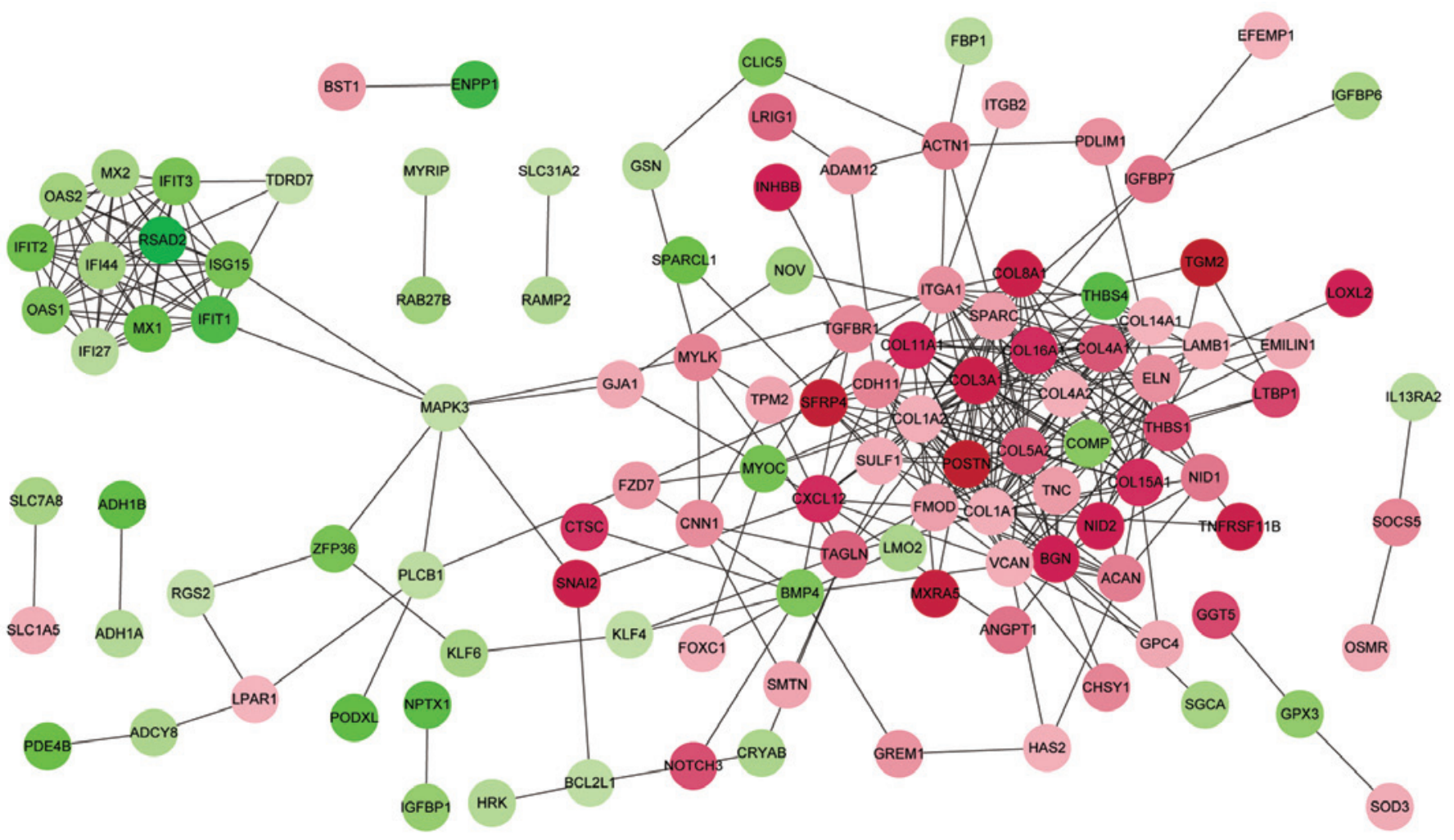

Figure 1. Protein-protein interaction network of DEGs. Red nodes represent upregulated genes in RA samples (downregulated in OA samples). Green nodes represent downregulated genes in RA samples. Color depth represents level of significance, with deeper colors indicating greater significance. DEG, differentially expressed gene; RA, rheumatoid arthritis; OA, osteoarthritis.
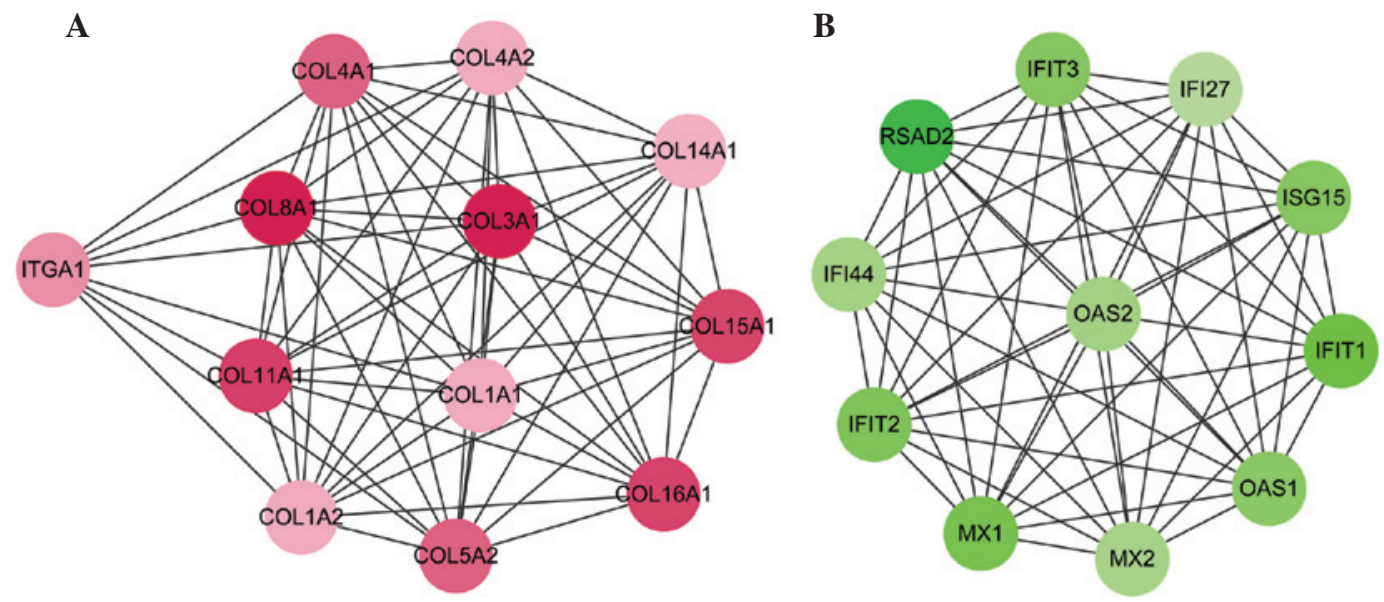

Figure 2. Protein-protein interaction network of DEGs in (A) Module 1 and (B) Module 2. Red nodes represent upregulated genes in RA samples (downregulated genes in OA samples). Green nodes represent downregulated genes in RA samples. Color depth represents level of significance, with deeper colors indicating greater significance. DEG, differentially expressed gene; RA, rheumatoid arthritis; OA, osteoarthritis.

Module 1, with the highest node score (11.455), consisted of 12 nodes and 63 edges, in which upregulated genes, including COL3A1, COL4A1, COL1A1 and COL11A1 were included (Fig. 2A). Module 2 (node score=11) consisted of 11 nodes and 55 edges, in which the downregulated genes were included (Fig. 2B). The downregulated genes in module 2 included radical S-adenosyl methionine domain containing 2 (RSAD2), 2'-5'-oligoadenylate synthetase 2 (OAS2), myxovirus (influenza virus) resistance 1 (MX1) and ISG15 ubiquitin-like modifier (ISG15).
In addition, functional enrichment analyses for DEGs in the functional modules were performed. Module 1 was predominantly associated with the regulation of cell proliferation, response to wounding and wound healing (Table IV). Module 2 was predominantly associated with immune functions, including the response to viruses, defense response and immune response (Table IV). The significantly enriched pathways of module 1 were ECM-receptor interaction and focal adhesion, whereas, no pathways were significant for module 2 (Table IV). 
Table IV. The significantly enriched GO biological progress terms for modules.

\begin{tabular}{clcc}
\hline GO term & \multicolumn{1}{c}{ Description } & No. enriched genes & P-value \\
\hline $\begin{array}{c}\text { Module } 1 \\
0042127\end{array}$ & Regulation of cell proliferation & 12 & 10 \\
0009611 & Response to wounding & 7 & $6.47 \times 10^{-10}$ \\
0042060 & Wound healing & 7 & $1.98 \times 10^{-9}$ \\
0001666 & Response to hypoxia & 7 & $3.28 \times 10^{-7}$ \\
0070482 & Response to oxygen levels & 9 & $4.70 \times 10^{-7}$ \\
0009725 & Response to hormone stimulus & 6 & $4.75 \times 10^{-7}$ \\
0031099 & Regeneration & 9 & $8.12 \times 10^{-7}$ \\
0009719 & Response to endogenous stimulus & 8 & $1.15 \times 10^{-6}$ \\
0008284 & Positive regulation of cell proliferation & 6 & $1.20 \times 10^{-6}$ \\
0001817 & Regulation of cytokine production & & $3.26 \times 10^{-6}$ \\
Module 2 & & 5 & $3.91 \times 10^{-8}$ \\
0009615 & Response to virus & $3.74 \times 10^{-2}$ \\
0006952 & Defense response & $3.40 \times 10^{-2}$ \\
0006955 & Immune response & 3 & 3 \\
\hline
\end{tabular}

GO, gene ontology.

Table V. Significantly enriched KEGG pathways for modules.

\begin{tabular}{llrl}
\hline Term & \multicolumn{1}{c}{ Description } & Count & P-value \\
\hline Module 1 & & & \\
hsa04512 & ECM-receptor interaction & 8 & $2.61 \mathrm{E}-13$ \\
hsa04510 & Focal adhesion & 8 & $1.36 \mathrm{E}-10$ \\
\hline
\end{tabular}

No significantly enriched pathways were identified for Module 2. Description represents the name of KEGG pathways and count represents the number of enriched genes. KEGG, Kyoto Encyclopedia of Genes and Genomes.

\section{Discussion}

There is a lack of effective treatments for RA and OA, and exploring the gene signatures associated with the diseases may elucidate the molecular pathogenesis and provide opportunities for biomarker development. The results of the present study demonstrated that the significant DEGs of module 1, including COL3A1, COL4A1, COL1A1 and COL11A1, were predominantly enriched in the ECM-receptor interaction and focal adhesion pathways. Additionally, the DEGs of module 2, including RSAD2, OAS2, MX1 and ISG15, were predominantly associated with immune responses. All these DEGs and pathways may be important mechanisms in the development of $\mathrm{RA}$ and $\mathrm{OA}$.

In a previous study, Olex et al (28) identified the ECM-receptor interaction pathway to be an important signaling and metabolic pathway during the progression of OA. Koelling et al (29) also demonstrated that several dysregulated genes in OA samples were associated with ECM-receptor interactions and focal adhesions. In the present study, DEGs, including COL3A1, COL4A1, COL1A1 and COL11A1, were predominantly enriched in the ECM-receptor interaction and focal adhesion pathways. It can, therefore, be speculated that the these pathways may contribute to OA progression. Additionally, COL3A1 is a gene important for cartilage function, and its expression was previously observed to be correlated with the radiographic severity of canine elbow OA (30). Cui et al (31) demonstrated that COL3A1 expression was enriched in the focal adhesion pathway, which may suggest a molecular mechanism of OA. Furthermore, COL4A1 was previously identified as an OA-associated gene involved in the development of the disease (32). Gene expression analysis also indicated that COL1A1 was dysregulated in TGF- $\beta$-stimulated OA samples (33). COL11A1 was demonstrated to be an OA susceptibility gene in human joint tissues and is important in the development of this degenerative musculoskeletal disease (34). Additionally, a previous investigation demonstrated that multiple collagen genes (COL1A1, COL2A1, COL3A1 and COL4A1) were associated with the progression of OA (28). Collagen derivatives are candidates for disease-modifying OA drugs and are marketed as having therapeutic effects on reducing the symptoms of OA (35). Therefore, it is speculated that these collagens may be important gene signatures of OA and have implications in the progression of this disease through effects on ECM-receptor interactions and focal adhesions.

Furthermore, the innate immune response is understood to cause inflammation and joint destruction in RA, and a 
genome-wide association study has demonstrated that immune regulatory factors underlie this disease (36). Additionally, the potential of Tank-binding kinase 1 as a therapeutic target in RA suggests that there is an association between the synoviocyte innate immune responses and RA development (37). McInnes and Schett (1) also investigated the importance of immune responses in the pathogenesis of RA. In the current study, GO functions associated with the immune response were enriched. Thus, the results of the present study support previous findings and suggest that immune responses may contribute to RA development.

Additionally, functional enrichment analysis performed in the present study demonstrated that DEGs in RA samples, including RSAD2, OAS2, MX1 and ISG15, were significantly enriched in the immune response pathway. RSAD2 is a type I interferon (IFN) response gene, and has been used in the clinic for the prediction of RA development $(38,39)$. OAS2 is involved in the IFN $\beta$ signaling pathway, and investigation of core and periphery interaction structures previously identified it as a candidate gene associated with RA (16). MX1 is also an IFN response gene and was previously demonstrated to be correlated with disease activity in fibroblast cells of RA synovial tissue (40). ISG15 sensitizes the IFN-activated JAK-STAT pathway, which is established to be important in RA development (41). Additionally, type I IFN has been demonstrated to enhance immune responses in vivo and acts as a signal linking innate and adaptive immunity (42). Smith et al (43) demonstrated that, as type I IFN signatures, RSAD2, OAS2 and MX1 may be important for predicting treatment response in RA. Therefore, the DEGs identified in the present study may be crucial gene signatures for elucidating the molecular pathogenesis of RA.

In conclusion, the results of the current study indicate that RSAD2, OAS2, MX1 and ISG15 may be RA gene signatures, and may be associated with RA development via effects on immune responses. COL3A1, COL4A1, COL1A1 and COL11A1 may be important gene signatures contributing to OA development via involvement in ECM-receptor interactions and focal adhesion. The present findings aid the clarification of the molecular mechanisms of RA and OA. However, the sample size used in the current study was small. Additional experiments, including reverse transcription-quantitative polymerase chain reaction and western blot analysis, were not performed to confirm the mRNA and protein expression levels of these gene signatures. Thus, further studies are required to investigate the potential clinical applications of these gene signatures.

\section{Acknowledgements}

The current study was supported by 5010 Clinical Research Project of Sun Yat-sen University (no. 2010005) and the National Natural Science Foundation of China (nos. 81371941 and 81301558).

\section{References}

1. McInnes IB and Schett G: The pathogenesis of rheumatoid arthritis. N Engl J Med 365: 2205-2219, 2011.

2. Wong JB, Ramey DR and Singh G: Long-term morbidity, mortality, and economics of rheumatoid arthritis. Arthritis Rheum 44: 2746-2749, 2001.
3. Loeser RF, Goldring SR, Scanzello CR and Goldring MB: Osteoarthritis: A disease of the joint as an organ. Arthritis Rheum 64: 1697-1707, 2012.

4. Wang HJ, Yu CL, Kishi H, Motoki K, Mao ZB and Muraguchi A: Suppression of experimental osteoarthritis by adenovirus-mediated double gene transfer. Chin Med J (Engl) 119: 1365-1373, 2006.

5. Huber R, Hummert C, Gausmann U, Pohlers D, Koczan D, Guthke R and Kinne RW: Identification of intra-group, inter-individual, and gene-specific variances in mRNA expression profiles in the rheumatoid arthritis synovial membrane. Arthritis Res Ther 10: R98, 2008.

6. van den Berg WB: Osteoarthritis year 2010 in review: Pathomechanisms. Osteoarthritis Cartilage 19: 338-341, 2011.

7. Knevel R, Gröndal G, Huizinga TW, Visser AW, Jónsson H, Víkingsson A, Geirsson AJ, Steinsson K and van der Helm-van Mil AH: Genetic predisposition of the severity of joint destruction in rheumatoid arthritis: A population-based study. Ann Rheum Dis 71: 707-709, 2012.

8. Bian Q, Wang YJ, Liu SF and Li YP: Osteoarthritis: Genetic factors, animal models, mechanisms, and therapies. Front Biosci (Elite Ed) 4: 74-100, 2012.

9. Bax M, van Heemst J, Huizinga TW and Toes RE: Genetics of rheumatoid arthritis: What have we learned? Immunogenetics 63: 459-466, 2011.

10. Sun Y, Caplazi P, Zhang J, Mazloom A, Kummerfeld S, Quinones G, Senger K, Lesch J, Peng I, Sebrell A, et al: PILR $\alpha$ negatively regulates mouse inflammatory arthritis. J Immunol 193: 860-870, 2014.

11. Castaño Betancourt MC, Cailotto F, Kerkhof HJ, Cornelis FM, Doherty SA, Hart DJ, Hofman A, Luyten FP, Maciewicz RA, Mangino M, et al: Genome-wide association and functional studies identify the DOT1L gene to be involved in cartilage thickness and hip osteoarthritis. Proc Natl Acad Sci USA 109: 8218-8223, 2012.

12. Valdes AM, Spector TD, Tamm A, Kisand K, Doherty SA, Dennison EM, Mangino M, Tamm A, Kerna I, Hart DJ, et al: Genetic variation in the SMAD3 gene is associated with hip and knee osteoarthritis. Arthritis Rheum 62: 2347-2352, 2010.

13. Yoshida S, Arakawa F, Higuchi F, Ishibashi Y, Goto M, Sugita Y, Nomura Y, Niino D, Shimizu K, Aoki R, et al: Gene expression analysis of rheumatoid arthritis synovial lining regions by cDNA microarray combined with laser microdissection: Up-regulation of inflammation-associated STAT1, IRF1, CXCL9, CXCL10, and CCL5. Scand J Rheumatol 41: 170-179, 2012.

14. Chanalaris A: Identification of a gene signature for osteoarthritis by comparing microarray data from rodent and human cartilage studies. Osteoarthritis Cartilage 22: S348-S349, 2014.

15. Lu QY, Han QH, Li X, Li ZC, Pan YT, Liu L and Fu QG: Analysis of differentially expressed genes between rheumatoid arthritis and osteoarthritis based on the gene co-expression network. Mol Med Rep 10: 119-124, 2014.

16. Singh S, Snijesh V and Vennila JJ: Rheumatoid arthritis candidate genes identification by investigating core and periphery interaction structures. In: Computational Intelligence in Medical Informatics. Muppalaneni NB and Gunjan VK (eds). 1st edition. Springer-Verlag, Singapore, pp87-96, 2015.

17. Pohlers D, Beyer A, Koczan D, Wilhelm T, Thiesen HJ and Kinne RW: Constitutive upregulation of the transforming growth factor-beta pathway in rheumatoid arthritis synovial fibroblasts. Arthritis Res Ther 9: R59, 2007.

18. Gautier L, Cope L, Bolstad BM and Irizarry RA: Affy - analysis of Affymetrix GeneChip data at the probe level. Bioinformatics 20: 307-315, 2004.

19. Irizarry RA, Hobbs B, Collin F, Beazer-Barclay YD, Antonellis KJ, Scherf U and Speed TP: Exploration, normalization, and summaries of high density oligonucleotide array probe level data. Biostatistics 4: 249-264, 2003.

20. Smyth GK: Limma: Linear models for microarray data. In: Bioinformatics and Computational Biology Solutions Using $\mathrm{R}$ and Bioconductor. Gentleman R, Carey V, Huber W, Irizarry R and Dudoit S (eds). Springer-Verlag, New York, NY, pp397-420, 2005.

21. Ashburner M, Ball CA, Blake JA, Botstein D, Butler H, Cherry JM, Davis AP, Dolinski K, Dwight SS, Eppig JT, et al: Gene Ontology: Tool for the unification of biology. Nat Genet 25: 25-29, 2000.

22. Kanehisa M and Goto S: KEGG: Kyoto encyclopedia of genes and genomes. Nucleic Acids Research 28: 27-30, 2000. 
23. Huang DW, Sherman BT, Tan Q, Collins JR, Alvord WG, Roayaei J, Stephens R, Baseler MW, Lane HC and Lempicki RA: The DAVID Gene Functional Classification Tool: A novel biological module-centric algorithm to functionally analyze large gene lists. Genome Biol 8: R183, 2007.

24. von Mering C, Huynen M, Jaeggi D, Schmidt S, Bork P and Snel B: STRING: A database of predicted functional associations between proteins. Nucleic Acids Res 31: 258-261, 2003.

25 . He $X$ and Zhang J: Why do hubs tend to be essential in protein networks? PLoS Genet 2: e88, 2006

26. Shannon P, Markiel A, Ozier O, Baliga NS, Wang JT, Ramage D, Amin N, Schwikowski B and Ideker T: Cytoscape: A software environment for integrated models of biomolecular interaction networks. Genome Res 13: 2498-2504, 2003.

27. Bader GD and Hogue CW: An automated method for finding molecular complexes in large protein interaction networks. BMC Bioinformatics 4: 2, 2003.

28. Olex AL, Turkett WH, Fetrow JS and Loeser RF: Integration of gene expression data with network-based analysis to identify signaling and metabolic pathways regulated during the development of osteoarthritis. Gene 542: 38-45, 2014.

29. Koelling S, Kruegel J, Irmer M, Path JR, Sadowski B, Miro X and Miosge N: Migratory chondrogenic progenitor cells from repair tissue during the later stages of human osteoarthritis. Cell Stem Cell 4: 324-335, 2009.

30. Clements DN, Fitzpatrick N, Carter SD and Day PJ: Cartilage gene expression correlates with radiographic severity of canine elbow osteoarthritis. Vet J 179: 211-218, 2009.

31. Cui S, Zhang X, Hai S, Lu H, Chen Y, Li C, Tong P, Lu F and Yuan Z: Molecular mechanisms of osteoarthritis using gene microarrays. Acta Histochemica 117; 62-68, 2015.

32. Rao ZT, Wang SQ and Wang JQ: Exploring the osteoarthritis-related genes by gene expression analysis. Eur Rev Med Pharmacol Sci 18: 3056-3062, 2014.

33. Remst DF, Blom AB, Vitters EL, Bank RA, van den Berg WB, Blaney Davidson EN and Kraan PM: Gene expression analysis of murine and human osteoarthritis synovium reveals elevation of transforming growth factor $\beta$-responsive genes in osteoarthritis-related fibrosis. Arthritis Rheum 66: 647-656, 2014.
34. Raine EV, Dodd AW, Reynard LN and Loughlin J: Allelic expression analysis of the osteoarthritis susceptibility gene COL11A1 in human joint tissues. BMC Musculoskelet Disord 14: 85, 2013.

35. Van Vijven JP, Luijsterburg PA, Verhagen AP, Van Osch GJ, Kloppenburg M and Bierma-Zeinstra SM: Symptomatic and chondroprotective treatment with collagen derivatives in osteoarthritis: A systematic review. Osteoarthritis Cartilage 20: 809-821, 2012.

36. Burton PR, Clayton DG, Cardon LR, Craddock N, Deloukas P, Duncanson A, Kwiatkowski DP, McCarthy MI, Ouwehand WH, Samani NJ, et al: Genome-wide association study of 14,000 cases of seven common diseases and 3,000 shared controls. Nature 447: 661-678, 2007.

37. Hammaker D, Boyle DL and Firestein GS: Synoviocyte innate immune responses: TANK-binding kinase-1 as a potential therapeutic target in rheumatoid arthritis. Rheumatology (Oxford) 51: 610-618, 2012

38. Lübbers J, Brink M, van de Stadt LA, Vosslamber S, Wesseling JG, van Schaardenburg D, Rantapää-Dahlqvist S and Verweij CL: The type I IFN signature as a biomarker of preclinical rheumatoid arthritis. Arthritis Res Ther 72: 776-780, 2013

39. Raterman HG, Vosslamber S, de Ridder S, Nurmohamed MT, Lems WF, Boers M, van de Wiel M, Dijkmans BA, Verweij CL and Voskuyl AE: The interferon type I signature towards prediction of non-response to rituximab in rheumatoid arthritis patients. Arthritis Res Ther 4: R95, 2012.

40. Galligan CL, Baig E, Bykerk V, Keystone EC and Fish EN: Distinctive gene expression signatures in rheumatoid arthritis synovial tissue fibroblast cells: Correlates with disease activity. Genes Immun 8: 480-491, 2007.

41. Boyle DL, Soma K, Hodge J, Kavanaugh A, Mandel D, Mease P, Shurmur R, Singhal AK, Wei N, Rosengren S, et al: The JAK inhibitor tofacitinib suppresses synovial JAK1-STAT signalling in rheumatoid arthritis. Ann Rheum Dis 74: 1311-1316, 2015.

42. Le Bon A and Tough DF: Links between innate and adaptive immunity via type I interferon. Curr Opin Immunol 14: 432-436, 2002.

43. Smith SL, Plant D, Eyre S and Barton A: The potential use of expression profiling: Implications for predicting treatment response in rheumatoid arthritis. Ann Rheum Dis 72: 1118-1124, 2013. 